\title{
Heuristic Channel Estimation Based on Compressive Sensing in LTE Downlink Channel
}

\author{
Lin Wan ${ }^{1}$, Min Wang ${ }^{1,2}$, Lifeng Su${ }^{1}$, Jun $\mathrm{Wu}^{1}$ \\ ${ }^{1}$ College of Electronics and Information Engineering, Tongji University, Shanghai, China \\ ${ }^{2}$ School of Mathematics and Computer Science, Gannan Normal University, Ganzhou, China \\ Email: 1131662@tongji.edu.cn,2011mwangcs@tongji.edu.cn,sulifeng@tongji.edu.cn,wujun@tongji.edu.cn
}

Received June, 2013

\begin{abstract}
Pilot-assisted channel estimation has been investigated to improve the performance of OFDM based LTE systems. LS and MMSE method do not perform excellently because they do not consider the inherent sparse feature of wireless channel. The sparse feature of channel impulse response satisfies the requirement of using compressive sensing (CS) theory, which has recently gained much attention in signal processing. Result in the application of using compressive sensing to estimate fading channel. And it achieves a much better performance than that with traditional methods. In this paper, we propose heuristic channel estimation based on CS in LTE Downlink channel. According to the feature of recovery algorithm in CS, we design a modified pilot placement method. CS recovery algorithms for channel estimation don't consider the statistics character of channel. So we proposed an optimization method which combines the CS and noise reduction. First we get initial channel statistics obtained by LS. Let the channel statistics as the heuristic information input of CS recovery algorithm. Then we perform CS recovery algorithm to estimate channel. Simulation results show this approach significantly reduces the complexity of channel estimation and get a better mean square error (MSE) performance.
\end{abstract}

Keywords: Channel Estimation; Compressed Sensing; Sparse Channel; LTE; Noise Reduction

\section{Introduction}

OFDM modulation is widely used in LTE systems which suffer from time and frequency fading channels. However, the performance of wireless system relies heavily on the validity of OFDM channel estimation. Generally, we can achieve better performance through two approaches, e.g., pilot design and channel estimate methods. Several pilot-aided channel estimation schemes have been discussed in [1-4], and measured the performance in terms of bit error rate (BER) and symbol error rate (SER). Many channel estimation methods are proposed to improve the performance of wireless communication system. LS algorithm [1], as the simplest method, has very low complexity, but it is extremely sensitive to AWGN noise. The MMSE algorithm [2] yields much better performance than LS estimator. Its high complexity hinders the implementation practically because matrix inversion is needed each time [3]. Although the complexity of MMSE is reduced by deriving singular value decomposition techniques, it highly depends on the detail channel statistics [4].

Wireless channels in practice are typically sparse [5],

${ }^{*}$ This work is financially supported by NSFC General Program under contract No.61173041. with very few of channel impulse response is nonzero. Traditional channel estimation methods mentioned above don't take the sparse feature of the channel into account, this result in much more detection errors. Recently years, CS theory has gained much attention in mathematics and signal and communication processing. CS theory declares that signals can still be recovered exactly from sample signal at a rate much less than Nyquist sampling, if the signals are sparse [6]. Considering the sparse feature of impulse respond in wireless fading channel, it is possible that CS can be applied on wireless channel estimation. Zhang et al [7] proposed an optimization of orthogonal matching pursuit (OMP) algorithm, according to the channel characteristics, to increase the precision of the algorithm and reduce the complexity of the algorithm with the same number of iterations. But the approach still need much more compute while it cannot find the useful value in the index set, more iterations mean more complexity and calculating delay. ALL of the available methods used to channel estimation based on CS are sensitive to AWGN noise [8], especially at low signal to noise ratio SNRs, as well as sparse recovery algorithms. Inspired by this, we proposed a novel optimization method for OMP, which is that just need one iteration 
with the channel characteristics abstracted from LS estimation. From the LS estimation, we can find the useful value position and an approximate channel impulse respond, which help us to avoid iteration to find the position and errors location caused by noise at low SNRs.

This paper is organized as follows: Section II reviews compressive sensing theory. In addition, introduces the LTE downlink system model and the sparsely of the multi-path blocking fading channel. Section III gives modified pilot placement strategy, and proposes heuristic channel estimation algorithm based on CS. Section IV is the simulation and analysis of proposed channel estimation methods. Section V is conclusions.

\section{Background}

In this section, we first review compressive sensing theory. Then, we present the LTE framework model for multi-path block-fading channel.

\subsection{Compressive Sensing}

Compressive sensing is a revitalized theory in signal processing for sparse and compressible signals. Mathematically let signal $x \in R^{N}$ be a vector of $N \times 1$. Assuming signal $x$ is k-sparse under the orthogonal basis $\Psi$. Let $y \in R^{M}$ be a set of linear measurements of ${ }^{X}$ by using a measurement matrix $\Phi$, which is not related to the sparse basis $\Psi$.

$$
y=\Phi x=\Phi \Psi \theta=A^{c s} \theta
$$

where $\theta$ is the signal $x$ projected on the basis of $\Psi$. A is considered as the operator of CS to get observation of $\theta$. In order to be able to recover the original signal $\theta$ from the observation $\mathrm{y}$, CS must satisfy two essential conditions.

- For accurate reconstruction, the number of observation $\mathrm{M}$ should meet $K \leq c M / \log (N / M)$, where $\mathrm{c}$ is sufficiently small, $\mathrm{N}$ is the length of original signal.

- A should satisfy the restricted isometric property (RIP) [9], which is essential to CS recovery performance.

Signal recovery is the center of CS theory research, it can be considered as optimization problem. Under the sparsely of $\mathrm{k}$, it can get $\theta$ from (1) by solving $\ell_{0}$ norm minimization problem

$$
\min _{x \in R^{N}}\|x\|_{0} \quad \text { s.t. }\|y-A \theta\|_{2} \leq \sigma
$$

This is combination and NP hard problem. Donoho et al [10] proposed that it can be replaced by an optimization $\ell_{1}$-norm problem

$$
\min _{x \in R^{N}}\|x\|_{1} \quad \text { s.t. }\|y-A \theta\|_{2} \leq \sigma
$$

In recent years, a collection of sparse recovery algorithms has emerged with CS. Methods for solving CS re- covery problems can be roughly divided into two classes, including greedy algorithms and convex optimization algorithms. Orthogonal matching pursuit (OMP) algorithm is commonly employed to recovery signal. Tropp et al [11] proposed signal recovery from random measurements by OMP. Reference $[12,13]$ proposed subspace pursuit (SP) and compressive sampling matching pursuit (CoSaMP) to reconstruct signal, respectively.

\subsection{System Model}

In this paper, we only consider single-antenna case in LTE downlink system, because it is easy extending from single-antenna case to multi-antenna case. The system model is shown in Figure 1.

The complex baseband representation of a multi-path fading channel impulse response can be described by

$$
g(t)=\sum_{m=1}^{M} \alpha_{m} \delta\left(t-\tau_{m} T_{s}\right)
$$

where $T_{s}$ is the sampling interval, $\tau_{m}$ is the delay of path $\mathrm{m}, \alpha_{m}$ is a complex value that characterizes the attenuation and initial phase of path $\mathrm{m} . T_{G}$ denoted as the time length of a cyclic extension, and $0 \leq \tau_{m} T_{s} \leq T_{G}$.

Let $X=\left[x_{k}\right]^{T}$ and $Y=\left[y_{k}\right]^{T} \quad(\mathrm{k}=0, \cdots \cdots, \mathrm{N}-1)$ denote the frequency domain data at the transmitter and the frequency domain data at the receiver, respectively. Let $g=\left[g_{n}\right]^{T}, \quad n=\left[n_{n}\right]^{T}$ and $(\mathrm{n}=0, \cdots \cdots, \mathrm{N}-1)$, denote the time domain sampled channel Impulse response and zero-mean, white, complex Gaussian noise, respectively. Define the DFT matrix as,

$$
F=\left[\begin{array}{ccc}
W_{N}^{00} & \ldots & W_{N}^{0(N-1)} \\
\vdots & \ddots . . & \vdots \\
W_{N}^{(N-1) 0} & \cdots & W_{N}^{(N-1)(N-1)}
\end{array}\right]_{N \times N}
$$

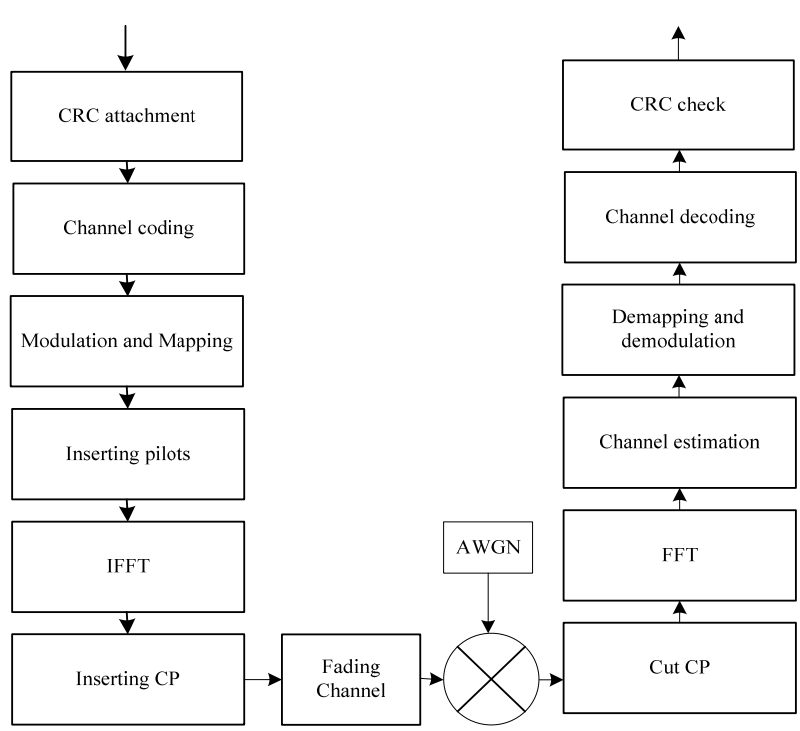

Figure 1. LTE downlink system model. 


$$
W_{N}^{n, k}=(1 / \sqrt{N}) e^{-j 2 \pi n k / N} .
$$

Furthermore, define $F=D F T_{N}(g)=F g$, the frequency domain channel impulse respond. NF is the frequency domain noise. NT is the time domain noise. Under the assumption that the interferences are completely eliminated, we can derive

$$
\begin{aligned}
Y & \left.=\operatorname{DFT}_{N}\left(\operatorname{IDFT}_{N}(X) * g\right)+N_{T}\right) \\
& =X F g+N_{F} \\
& =X H+N_{F}
\end{aligned}
$$

\section{Channel Estimation Based on Compressive Sensing}

In this section, we present our channel estimation scheme base on compressive sensing. We first give the modification of OFDM pilot placement in standard LTE system. Then, we give the derivation the principle of channel estimation combined with CS, and propose our optimization algorithm.

\subsection{Pilot Placement}

LTE downlink system applies the comb-type pilot placement. Single-antenna system pilot placement in one resource block is shown in Figure 2. The left figure depicts the pilot placement in standard LTE system. We note that the pilot placement of standard is uniform distribute in 2D plane, which composed by frequency domain and sub carriers. The channel responses of non pilot sub-carriers are estimated by interpolating neighboring pilot in sub channels. Suppose the block fading channel, the channel coefficients at the same subcarrier position within on block are all the same. So we can completely estimate the channel coefficients only by one-dimensional interpolation.

In order to achieve better performance of channel estimation by using CS signal recovery algorithms, we should modify the position of the pilot placement in 2D plane. There is a important factors to consider in pilot placement modification. That is the modification does a little change to the LTE system. So we must considering the LTE pilot placement and frame structure. [14] Express that CS-based channel estimation scheme can achieve better performance when use random pilot placement. In standard LTE pilot placement, every block has to provide four indexes to place pilot in twelve placements. So there is C4 12 combination. Through 1000 Monte carols simulation of random pilot placement; we find that the modified pilot placement, shown in the right figure of Figure 2, can achieve the best performance of channel estimation.

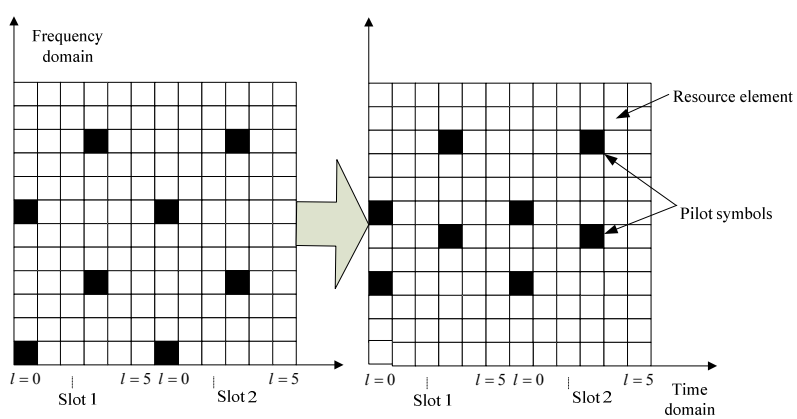

Figure 2. Pilot placement.

\subsection{Heuristic Channel Estimation Based on CS}

Traditional LS estimator minimizes the parameter by

$$
\hat{H}_{L S}=\arg \min \left|\left(Y_{p}-X_{p} \hat{H}\right)^{H}\left(Y_{p}-X_{p} \hat{H}\right)\right|
$$

where $(\bullet)^{H}$ means the conjugate transpose operation, $Y_{p}$ and $X_{p}$ denote as received and transmitted pilot value, respectively. LS estimator get initial estimation from pilots and interpolate to all the data position, we can get all the channel frequency respond. The pilot signal vector can be expressed as

$$
Y_{p}=X * \Psi_{s} F g+N_{F}
$$

where $\Psi_{s}$ is an identity matrix with dimensions $S \times N$.

$$
\Psi_{S}=\left[\begin{array}{ccccccccc}
0 & \cdots & 1 & \cdots & 0 & \cdots & 0 & \cdots & 0 \\
0 & \cdots & 0 & \cdots & 1 & \cdots & 0 & \cdots & 0 \\
\vdots & \cdots & \cdots & \cdots & \cdots & \cdots & \cdots & \cdots & \vdots \\
0 & \cdots & 0 & \cdots & 0 & \cdots & 1 & \cdots & 0
\end{array}\right]_{S \times N}
$$

Because the pilot has been known at the receiver, the impulse response of the channel at the pilot points is described as

$$
\begin{aligned}
\hat{H} & =Y_{p} / X_{p} \\
& =\Psi_{s} F g+N_{F} \\
& =\Phi g+N_{F}
\end{aligned}
$$

where $\Phi=\Psi_{S} F$ is called sensing matrix in the CS theory. Therefore, we can use the solution of CS methods for channel estimation. Once we recovery the channel impulse respond from CS algorithm, we can get the CS estimation.

$$
\hat{H}_{c s}=F F T(g)
$$

If the channel impulse response is k-sparse, the recovery algorithm needs $\mathrm{k}$ iteration at least. So the CS-based channel estimation algorithm still has very high complexity. Furthermore, the accuracy of estimation maybe much lower at low SNRs, result in the decrease of performance. 
In this subsection, we proposed a fast solution based on the combination of OMP method and channel statistics. The channel statistics can be obtained from LS estimation. First get initial estimation from pilots and then interpolate to all the data position, we can get all the channel frequency response. Evaluating the time domain estimation, we collect $\mathrm{k}$ of the most important taps, where the importance means the valve is large than others. And let $\mathrm{k}$ equals the length of a cyclic extension. We record the position of the collected taps and sort them in descend by module value. Here we called the collected set as the delay profile of channel, a useful index that used for OMP recovery. Here, the useful index is denoted by $\Theta$. Known the sparsely and the delay profile of channel, it means that we do not need iterate to find the possible position. With the $\Theta$, we can increase the precision and reduce the complexity of the algorithm to one.

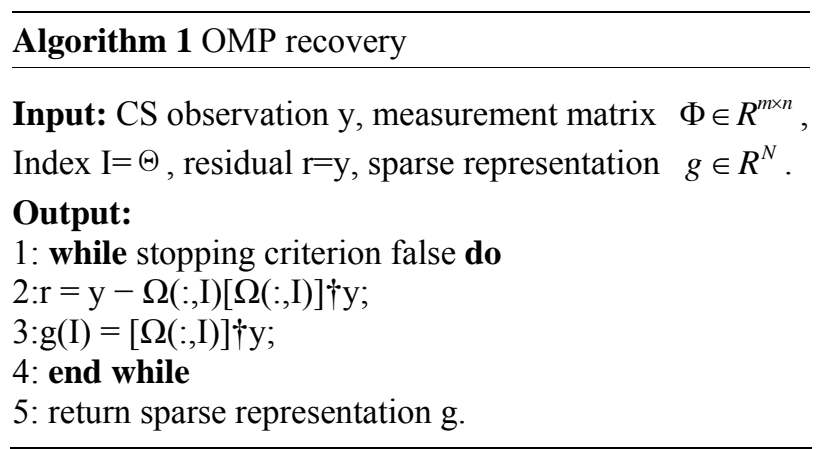

\section{Simulation and Results}

We performed computer simulations to verify the performance of the proposed methods applied to the LTE downlink system. We simulated a LTE downlink system with $20 \mathrm{MHz}$ bandwidth with $\mathrm{N}=2048$ sub-carriers. The channel model uses Vehicle model. Table 1 shows the profile of the channel parameters. Table II lists the simulation parameters of LTE systems. Under the same experimental condition, we compare the performance among LS, MMSE and proposed CS method by using normalized MSE and bit errors ratio (BER), respectively. The normalized MSE is defined as

$$
M S E=\frac{E\left|\sum i\right| H(i)-\left.\hat{H}(i)\right|^{2} \mid}{\left.E\left|\sum i\right| H(i)\right|^{2} \mid}
$$

Figure 3 depicts the comparison of the normalized MSE for standard pilot placement and optimized pilot placement. In this simulation, we perform LS and CS channel estimation algorithm for two pilot placement strategy respectively, and SNR range form $-5 \mathrm{~dB}$ to 30 $\mathrm{dB}$. It shows that two pilot strategies have the same performance at low SNRs. But in LS estimation, comparing to standard pilot placement, the performance of opti- mized pilot placement is slight worse at high SNRs. The reason of this phenomenon is that the uniform distribution averages the noise at LS interpolating, while the rand distribution does not have the ability when the noise is low enough at high SNRs.

Figure 4 shows the normalized MSE performance among LS, MMSE, CS and CS-optimized channel estimation. At low SNRs range, the CS estimation approximation effect is significantly better than LS, while much worse than MMSE. The reason is the noise. For the optimized CS estimation, its performance is much better than CS at lows SNRs, and as well at high SNRs. The reason is that the $\mathrm{CS}$ itself can find the right position as CS-OPT at high SNRs. However CS needs more complexity and iterations.

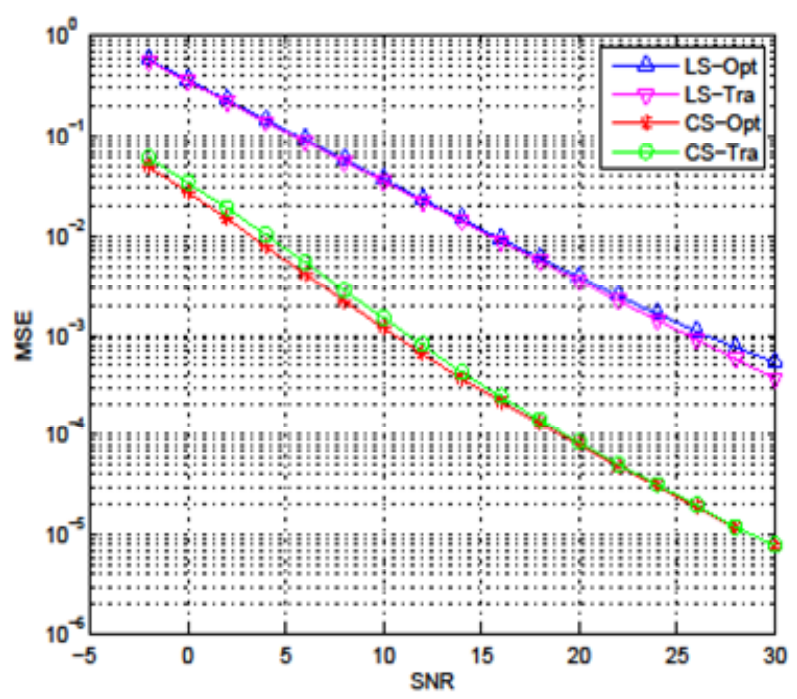

Figure 3. MSE Performance comparison between standard pilot placement and optimized pilot placement.

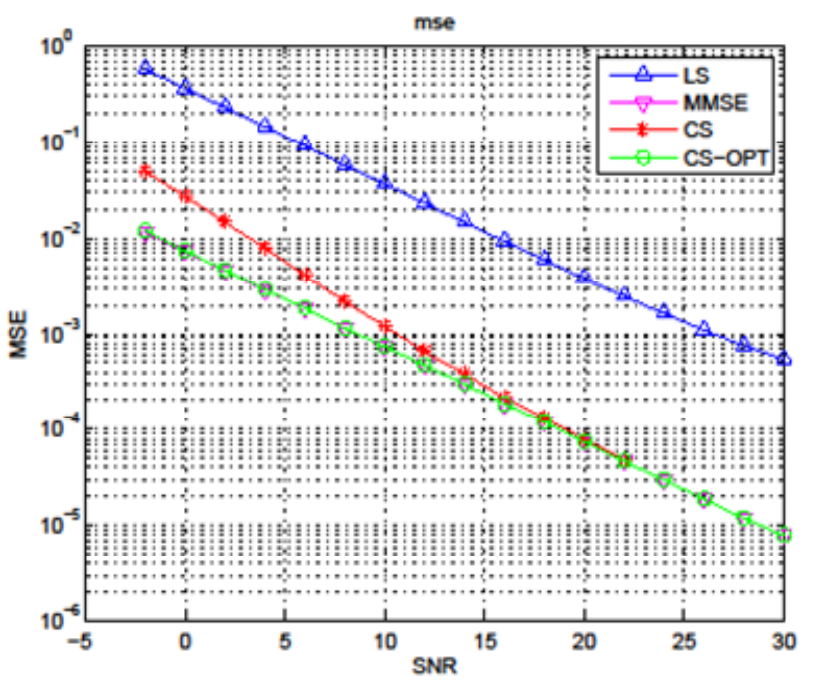

Figure 4. MSE Performance comparison among CS-OPT, CS, LS and MMSE channel estimation. 


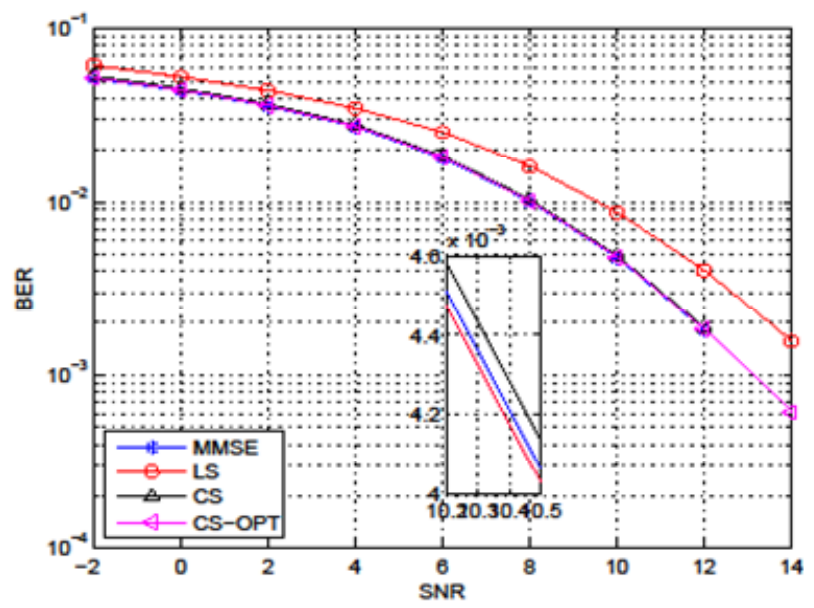

Figure 5. BER performance comparison among CS-OPT, CS, LS and MMSE channel estimation.

Figure 5 describes the BER performance of LS, MMSE, $\mathrm{CS}$ and CS-optimized channel estimation. It is seen that the BER performance of CS is much better than LS. It uses the sparse of the channel and exactly recovery some of channel impulse respond at low SNRs, and well-done impulse at high SNRs. The performance of LS is the worst because it does nothing about noise reduction and interpolation, which may cause much more errors. MMSE become the best one except Ideal along with the noise reduction by the channel autocorrelation. The optimized CS estimation. It is seen that at lows SNRs CSOPT is much better than CS method; because of at high SNRs the CS itself can find the right position the same as CS-OPT.

\section{Conclusions}

In this paper, we study the channel estimation method based on compressive sensing theory. We first present modified pilot placement strategy to suit CS channel estimation. We propose an optimization recovery algorithm based on OMP by using the channel statistics. Our simulation results demonstrate that the optimization of the CS-based channel estimation algorithm significantly promotes the performance compared to the traditional estimation and the reduction of complexity for CS recovery contributes to its implementation.

\section{REFERENCES}

[1] W. G. Jeon, K. H. Paik and Y. S. Cho, "An Efficient Channel Estimation Technique for Ofdm Systems with Transmitter Diversity," In Personal, Indoor and Mobile Radio Communications, 2000. PIMRC 2000, The 11th IEEE International Symposium on, Vol. 2, pp.
1246-1250.

[2] J.-J. van de Beek, O. Edfors, M. Sandell, S. K. Wilson, and P. Ola Borjesson, On Channel Estimation in Ofdm Systems, In Vehicular Technology Conference, 1995 IEEE 45th, Vol. 2, pp. 815-819.

[3] C. Mehlfuhrer, S. Caban and M. Rupp, "An Accurate and Low Complex Channel Estimator for Ofdm Wimax," In Communications, Control and Signal Processing, 2008, ISCCSP 2008, 3rd International Symposium on, pp. 922-926.

[4] M.-H. Hsieh and C.-H. Wei, "Channel Estimation for Ofdm Systems Based on Comb-type Pilot Arrangement in Frequency Selective Fading Channels," Consumer Electronics, IEEE Transactions on, Vol. 44, No. 1, pp. 217-225.

[5] A. M. Sayeed, Sparse Multipath Wireless Channels: Modeling and implications. 2006.

[6] E. J. Candes, J. Romberg and T. Tao, "Robust Uncertainty Principles: Exact Signal Reconstruction from Highly Incomplete Frequency Information," Information Theory, IEEE Transactions on, Vol. 52, No. 2, pp. 489-509.

[7] S. Zhang, J. Kang, Y. C. Song and N. N. Wang, "An Optimization for Channel Estimation Based on Compressed Channel Eensing," In Software Engineering, Artificial Intelligence, Networking and Parallel Distributed Computing (SNPD), 2012 13th ACIS International Conference on, pp. 597-602.

[8] H. Zamiri-Jafarian, M. J. Omidi and S. Pasupathy, Improved Channel Estimation Using Noise Reduction for Ofdm Systems. In Vehicular Technology Conference, 2003.VTC 2003-Spring, The 57th IEEE Semiannual, Vol. 2, pp. 1308-1312.

[9] E. J. Candes and T. Tao, "Decoding by Linear Programming. Information Theory," IEEE Transactions on, Vol. 51, No. 12, pp. 4203-4215.

[10] D. L. Donoho, "Compressed Sensing. Information Theory," IEEE Transactions on, Vol. 52, No. 4, pp. 1289-1306.

[11] J. A. Tropp and A. C. Gilbert, "Signal Recovery from Random Measurements via Orthogonal Matching Pursuit," Information Theory, IEEE Transactions on, Vol. 53, No. 12, pp. 4655-4666.

[12] W. Dai and O. Milenkovic, "Subspace Pursuit for Compressive Sensing Signal Reconstruction," Information Theory, IEEE Transactions on, Vol. 55, No. 5, pp. 2230-2249.

[13] D. Needell and J. A. Tropp. Cosamp: Iterative Signal Recovery from Incomplete and Inaccurate Samples. Technical Report, California Institute of Technology, Pasadena, 2008.

[14] C. H. Qi and L. N. Wu, "Optimized Pilot Placement for Sparse Channel Estimation in Ofdm Systems, Signal Processing Letters, IEEE, Vol. 18, No. 12, 2011, pp. 749-752. 